Macromolecular Chemistry: The second Century

March 22-26, 2020 Philadelphia, PA

Division of Physical Chemistry

Experimental \& Computational Approaches in Unraveling Mechanisms Of Amyloid Formation

\title{
Intrinsically Disordered Proteins share a common molecular mechanism in membranes damages: the lipid-chaperone hypothesis.
}

\author{
Carmelo La Rosa \\ University of Catania, Italy \\ Email: carmelo.larosa@unict.it
}


Recently, a phenomenological mode $\mathrm{l}^{1-6}$ based on experimental findings was proposed to simulate the transfer kinetics of a lipid-protein complex from water to the lipid-bilayer phase. We find that the water-soluble lipid-protein complex inserts into the membrane faster than the free protein due to the hydrophobic differences between the lipid-protein complex and the bare protein. This model is supported by several experiments carried out on human IAPP7.

Importantly, our results carried out on human IAPP offer a novel mechanistic explanation as to why bilayer thickness is inversely correlated to membrane damage induced by amyloid proteins. The increased bilayer damage seen for thin bilayers is caused by an increase of lipid-bound proteins causing faster insertion and membrane disruption as there are more free lipids in solution for the shorter acyl-chains (high CMC). Thus, it is not the thickness of the bilayer that is important in causing membrane damage, but rather the relative free lipid concentration in solution, coupled to the stability of the lipid-protein complex and its hydrophobicity.

Question: Are the results obtained an exclusive behavior of the human IAPP, or can they be extended to other IDPs?

An answer to this question can be given by investigating other IDPs. In this light, different amyloidogenic proteins, including $A \beta$ peptide and $\alpha$-synuclein was investigated. Further, we also investigated two non-amyloidogenic proteins, rat-IAPP and $\beta$-synuclein, as controls. 


\section{Remarks on human IAPP}

Self-assembled phospholipids into LUVs are in chemical equilibrium with free lipids in solution.

Free lipids in solution interacting with hIAPP form a stable lipid-protein complex

hAIPP interacting with LUVs containing high cme phospholipids $(\boldsymbol{\mu M})$ form pores and not fibrils.

hIAPP interacting with LUVs containing low $\mathbf{c m c}$ phospholipids (nM) form fibrils in aqueous phase and not pores

Free phospholipids, by forming a phospholipid-hIAPP complex act as chaperon and transport hIAPP into the bilayer

Question: is this molecular mechanism exclusively of hIAPP or it is a general mechanism common to Intrinsically Disordered Proteins?

In order to answer this question, we have investigated A $\beta 40, A \beta 42$, rIAPP, $\alpha$-synuclein and $\beta$ synuclein interacting with LUVs containing phospholipids with different cmc. 
Dye leakage and ThT assay of $A \beta(1-40)$ interacting with model membranes containing phospholipid having different CMC (phospholipid used; from PC 14 (1:0) to PC 20 (1:0) CMC PC 14 (1:0), PC 18 (1:0) and PC 20 (1:0): $1 \times 10^{-7} \mathrm{M}, 2 \times 10^{-8} \mathrm{M}$ and $6.3 \times 10^{-9} \mathrm{M}$ respectively.

A $\beta$ s form pore with a dimension of $1.8 \mathrm{~nm}$, so to determine leakage we use the couple $\mathrm{Ca}^{++} /$fura-2, instead carboxy-fluorescein.

Results: $A \beta(1-40)$ show the same behavior of hIAPP

In model membrane containing phospholipid with high CMC pore formation is enhanced and fibril formation is repressed

in model membrane containing phospholipid with low CMC pore formation is repressed and fibril formation is enhanced.

$\alpha$-synuclein show the same behavior of hIAPP and $A \beta(1-40)$. 
Is know that both rat IAPP and $\beta$-synuclein not form fibril and are not-toxic for cell. Here we report ThT assay and carboxy-fluorescein dye leakage of rat IAPP and $\beta$-synuclein. Results: al proteins concentration of $10 \mu \mathrm{M} \beta$-synuclein and rat IAPP not form fibril. Rat IAPP form pores according data from Raleigh lab ${ }^{9}$. Also rat IAPP in some condition is toxic. $\beta$-synuclein not form pores.

Experiments shown how pore formation of hIAPP, rIAPP and $\alpha$-synuclein not depend of bilayer protein-mismatch and bilayer thickness.

Addition of a relatively high concentration of free PC14 (1:0) (CMC $\left.1 \times 10^{-7}\right)$ to PC $22(1: 0)$ model membranes allows for the formation of the lipid-protein complex, promoting insertion into the bilayer, pore formation and increasing leakage.

NMR and CD measurements suggest the formation of lipid-protein complex.

On the increased hydrophobicity of lipid-protein complex respect bare protein

Link between our biophysical investigations and in vivo data: literature data ${ }^{10}$. Analyzing literature data emerge that possible source of free phospholipids should be lipid oxidation. 


\section{CONCLUSIONS}

\section{Free lipids form a stable complex with IDPs}

IDS interacting with membranes share common molecular mechanisms in pore and fibril formation

Lipid-Chaperone hypothesis is a more general molecular model that include both amyloid and oligomer hypothesis

Oxidized lipids are candidate to trigger Diabetes, Alzheimer and Parkinson 
